JOURNAL OF THE

AMERICAN MATHEMATICAL SOCIETY

Volume 22, Number 3, July 2009, Pages 797-819

S 0894-0347(08)00619-X

Article electronically published on September 16, 2008

\title{
ASYMMETRY OF NEAR-CRITICAL PERCOLATION INTERFACES
}

\author{
PIERRE NOLIN AND WENDELIN WERNER
}

\section{INTRODUCTION}

A short general overview. The goal of the present paper is to study some aspects of two-dimensional percolation near its critical point. Recall that the mathematical understanding and description of the percolation phase transition in the plane has improved a lot during the last decade, thanks to two new main ingredients: the derivation of conformal invariance of critical percolation on the triangular lattice by Smirnov [27] and the description of the scaling limits of interfaces of critical percolation via Schramm's SLE(6) process introduced in 25. This has allowed one to identify the critical exponents that had been predicted in the physics literature via conformal field theory, Coulomb gas methods or quantum gravity. One new feature of the SLE approach compared to the methods developed by physicists is to view interfaces as global random geometric objects and to go beyond the "correlation functions". This is a little bit like defining the infinitesimal generator of a Markov process and studying its trajectories instead of just looking at its finite-dimensional distributions. A by-product of this approach is the universality of certain random curves. For instance, one can prove that outer boundaries of scaling limits of percolation clusters, outer boundaries of planar Brownian loops or the conjectured scaling limits of self-avoiding random loops all have exactly the same distribution (see, e.g. 21, 31]). Such a strong statement had not been formulated in the physics literature, where the corresponding models are just said to belong to the same "universality classes".

The understanding of the large-scale properties of percolation at its critical point also enabled the description of "near-critical" models, thanks to Kesten's scaling relations [16]. This allowed one to to derive critical exponents that describe how certain quantities behave when the percolation parameter is close (but not equal) to its critical value. This is roughly speaking due to the fact that in the regime under consideration, the "large-scale connectivity properties" remain comparable to those of critical percolation. In the physics language, these near-critical models also belong to the same universality class as critical percolation.

The goal of the present paper is to study the global geometry of these nearcritical percolation interfaces. Our main result is that - despite the fact that their

Received by the editors December 4, 2007.

2000 Mathematics Subject Classification. Primary 60K35, 82B27, 82B43.

Key words and phrases. Near-critical percolation, off-critical regime, massive scaling limit, random fractal interfaces, SLE.

This research was supported by the Agence Nationale pour la Recherche under the grant ANR-06-BLAN-0058.

(c)2008 American Mathematical Society Reverts to public domain 28 years from publication 
"finite-dimensional marginals" are comparable - the scaling limits of near-critical percolation interfaces do intrinsically differ from SLE(6) curves. By looking at any portion of a sample of such a curve, one can almost surely detect the difference from an SLE curve. The asymmetry of a near-critical model remains visible in the scaling limit. This shows the existence of other "universal" random curves than SLE. It is worth noticing that in most real-life occurrences of critical phenomena, the interfaces that one observes are these near-critical ones and not the "exactly critical" ones.

Background: critical percolation and SLE. We shall study site percolation on the triangular planar lattice. Recall that this can be viewed as a random coloring of the hexagonal cells of a honeycomb lattice, where the color (black or white) of each cell is chosen independently of the others: each of these cells has a probability $p$ to be black and $1-p$ to be white, for some parameter $p$ between 0 and 1 . In percolation theory, one is interested in the connectivity properties of the set of black hexagons (or the set of white ones). They can be regrouped into maximal connected components called clusters. The phase transition for percolation on this lattice occurs at $p=1 / 2$. Often, it is described mathematically as follows, in terms of almost sure properties of percolation in the infinite lattice: when $p<1 / 2$, there exists with probability 1 no infinite cluster of black sites (subcritical regime) and an infinite cluster of white sites, and conversely when $p>1 / 2$, there is an infinite cluster of black sites (supercritical regime) but no infinite cluster of white sites. In the critical case where $p=1 / 2$, there exists neither an infinite white cluster nor an infinite black cluster - but if one takes a finite large piece $\Lambda$ of the lattice, one will see white and black clusters of size comparable to that of $\Lambda$. See, e.g. 14, 15] for an introduction to percolation.

A lot of progress has recently been made in the understanding of the large-scale behavior of critical percolation: in particular, Smirnov [27] proved conformal invariance of the connection probabilities, which allowed one to make the link [27, 4, 28] with the Schramm-Loewner Evolution (SLE) with parameter 6 introduced in 25] and to use the SLE technology and computations [18, 19] to derive further properties of critical percolation, such as the value of some critical exponents, describing the asymptotic behavior of the probabilities of certain exceptional events (arm exponents) 20, 29]. We refer to 32] for a survey.

One precise relation to SLE is as follows: we consider the large equilateral triangle $T_{N}$ with even side length $N$ on the triangular grid such that the middle of the bottom part is the origin and the top point is the point at distance $\sqrt{3} N / 2$ above the origin. We decide to color all cells on the boundary of the triangle, in white if their $x$-coordinate is positive and in black if their $x$-coordinate is negative, and we perform critical percolation in the inside of $T_{N}$. Then, we consider the interface $\gamma^{N}$ (viewed as a path on the hexagonal lattice dual to the triangular lattice) between the set of black sites attached to the left part of the triangle and the set of white sites connected to the right part of the triangle (see Figure 1). When $N \rightarrow \infty$, the law of the rescaled interface $\Gamma_{N}:=\gamma^{N} / N$ converges (in an appropriate topology) to that of the $\operatorname{SLE}(6)$ process from $(0,0)$ to $(0, \sqrt{3} / 2)$ in the equilateral triangle with unit side length. See [5, 28, 32] for details, and, e.g. 17] for an introduction to SLE. Thanks to this convergence result, one is able to deduce the properties of critical percolation from the properties of SLE. For instance (and we shall come back to this later), one can prove that the typical number of steps of the path $\gamma^{N}$ is 


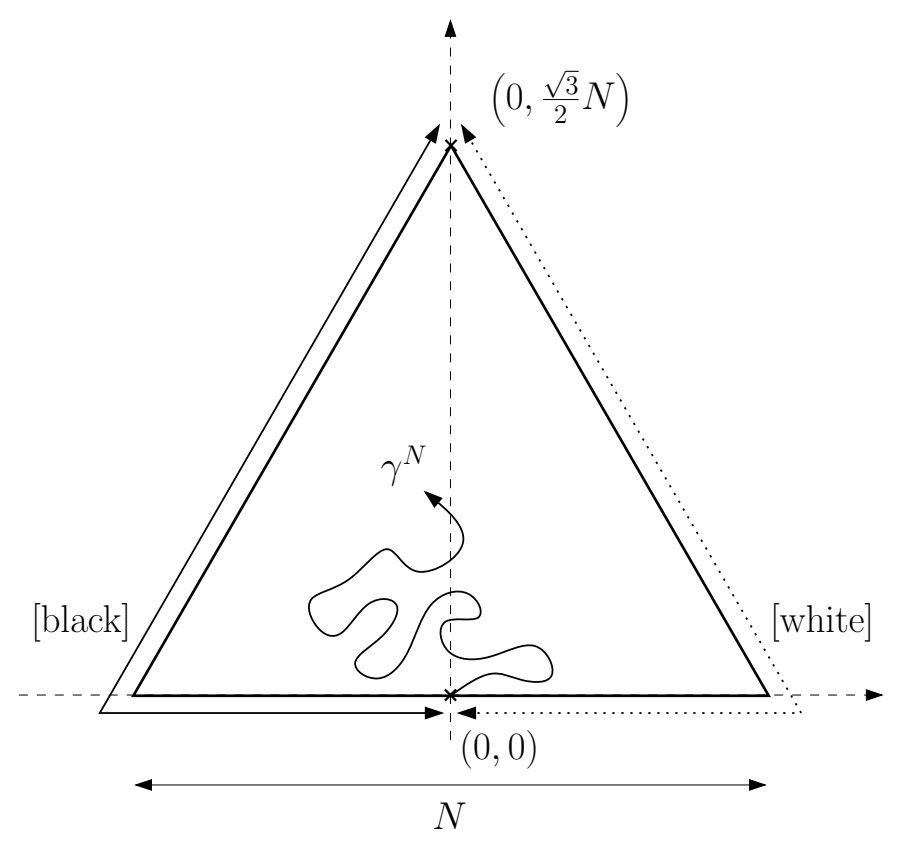

Figure 1 . The triangle $T_{N}$ and the interface $\gamma^{N}$ (sketch).

of the order $N^{7 / 4}$ (more precisely, for each $\epsilon>0$, the probability that the number of steps is between $N^{7 / 4-\epsilon}$ and $N^{7 / 4+\epsilon}$ goes to 1 as $N \rightarrow \infty$ ).

Background: near-critical percolation. Understanding the behavior of critical percolation also allows one to derive some properties of percolation when the parameter $p$ is very close to $1 / 2$. These are the scaling (and hyperscaling) relations that were first developed in the physics literature (the names of Fischer, Kadanoff and Wilson are often associated to these ideas) and later rigorously derived in the case of percolation by Kesten [16] (see also [23, 32]). Before explaining these relations in a little more detail, let us first make a trivial observation to help the newcomer to get a feeling of what goes on.

Recall that it is easy to couple realizations of percolation for all values of $p$ on the same probability space in an increasing manner, in such a way that if a site is black for the realization $w_{p}$ (that follows the law $P_{p}$ of percolation with parameter $p)$, then it is also black for all $w_{p^{\prime}}$ with $p^{\prime}>p$. To do this, one can for instance introduce an i.i.d. family of uniform random variables $U_{x}$ on $[0,1]$, where $x$ spans the set of all sites on the lattice, and declare that $x$ is black for $w_{p}$ if $U_{x} \leq p$ and white otherwise. For each given $x$, the probability that the color of $x$ is not the same for $w_{p}$ as for $w_{p^{\prime}}$ is thus $\left|p-p^{\prime}\right|$.

Suppose now that we consider the percolation interface in the large equilateral triangle as before, but choose $p=p(N)$ in such a way that $p-1 / 2=o\left(N^{-7 / 4-\epsilon}\right)$. Then, one can couple it with a critical percolation interface (i.e. with the case when $p=1 / 2$ exactly) in the same triangle in such a way that the two paths are identical except on an event of probability that goes to 0 as $N \rightarrow \infty$ (this is just because the probability that the color of one of the neighbors of the interface differs between 
$w_{p}$ and $w_{1 / 2}$ is bounded by $|p-1 / 2|$ times the number of neighbors of the interface - and we have just seen that this number is $\left.o\left(N^{7 / 4+\epsilon}\right)\right)$. Hence, if one lets $N$ go to infinity, the scaling limit of the interface is still an $\operatorname{SLE}(6)$. In particular, the probability that the interface $\Gamma_{N}$ hits the right side of the triangle before the left side of the triangle - let us call $R(p, N)$ this probability - goes to $1 / 2$ as $N \rightarrow \infty$.

On the other hand, for any fixed $\epsilon \in(0,1 / 2)$, one can define

$$
p^{*}(N)=p^{*}(N, \epsilon):=\inf \{p: R(p, N)>1 / 2+\epsilon\} .
$$

For this choice of $p=p^{*}(N)$, if one looks at the possible limiting behavior of $\Gamma_{N}$, it is clear that the law cannot be exactly that of an $\operatorname{SLE}(6)$ anymore because it will hit the right side of the triangle before the left one with probability at least $1 / 2+\epsilon$ (whereas this probability is $1 / 2$ for $\operatorname{SLE}(6)$ ). It is therefore natural to ask what can happen to the scaling limit of this curve when $N \rightarrow \infty$ in this regime and to see how it is related (or not) to $\operatorname{SLE}(6)$.

One can equivalently define the so-called correlation length $L(p)=L(p, \epsilon)$ in such a way that $p^{*}(L(p), \epsilon) \simeq p$. In other words, for $p>1 / 2$,

$$
L(p)=L(p, \epsilon):=\inf \{N: R(p, N)>1 / 2+\epsilon\}
$$

(note that for $p>1 / 2$ fixed, $R(p, N) \rightarrow 1$ as $N \rightarrow \infty$ so that $L(p)<\infty$ ). Kesten [16] has shown that it is possible to deduce from the arm exponents of critical percolation the behavior of $L(p)$ as $p \rightarrow 1 / 2$. This derivation relies on the "four-arm exponent": in the standard coupling, the event corresponding to $R(p, N)$ appears exactly when a "pivotal" site is flipped (we will discuss this is more detail in the next section). Combining Kesten's results with the exponents computed using SLE, one gets 29] that

$$
L(p)=(p-1 / 2)^{-4 / 3+o(1)}
$$

when $p \rightarrow 1 / 2^{+}$, for any fixed choice of $\epsilon \in(0,1 / 2)$. This is (see 16, 23, 32, ) a crucial step in the rigorous proof of the fact that the "density" $\theta(p)$ of the infinite cluster for percolation with parameter $p$ decays like $(p-1 / 2)^{5 / 36+o(1)}$ as $p$ decays to $1 / 2^{+}$.

We shall see (and this is quite easy) that in order to get a non-trivial limit for $\Gamma_{N}$ (i.e. neither an $\operatorname{SLE}(6)$ nor a path that just sticks to the boundary of $T$ ) one has to take $p(N)$ in such a way that $N$ is of the order of the correlation length $L(p)$, i.e. that $p(N) \in\left[p^{*}(N, \epsilon), p^{*}\left(N, \epsilon^{\prime}\right)\right]$ for some $\epsilon<\epsilon^{\prime}$. The terminology to describe this type of regime depends on the circumstances and the papers: near-critical, offcritical, massive scaling (in the Free Field context). It is also very closely related to the scaling and hyperscaling relations. Anyway, it has been the subject of numerous and interesting works; see, e.g. [1, 8, 3, 10] and the references therein.

One general rule behind the derivation of these results is that as long as $n \leq L(p)$, estimates remains roughly the same as at criticality. For example, the probabilities of the existence of crossings of annuli for near-critical percolation are bounded by a constant times those at criticality (see more details in the next section). This implies that the exponents describing critical percolation also describe off-critical percolation and lead to Kesten's scaling relations. In [6, 7], Camia, Fontes and Newman has suggested that the scaling limit of the near-critical picture could be obtained from that of the critical picture by just opening a Poissonian family of "pivotal" points. We understand that Garban, Pete and Schramm [13 have succeeded in proving this fact. A consequence would be that the subsequential limits that we are discussing in the present paper are in fact limits, and that they are all 


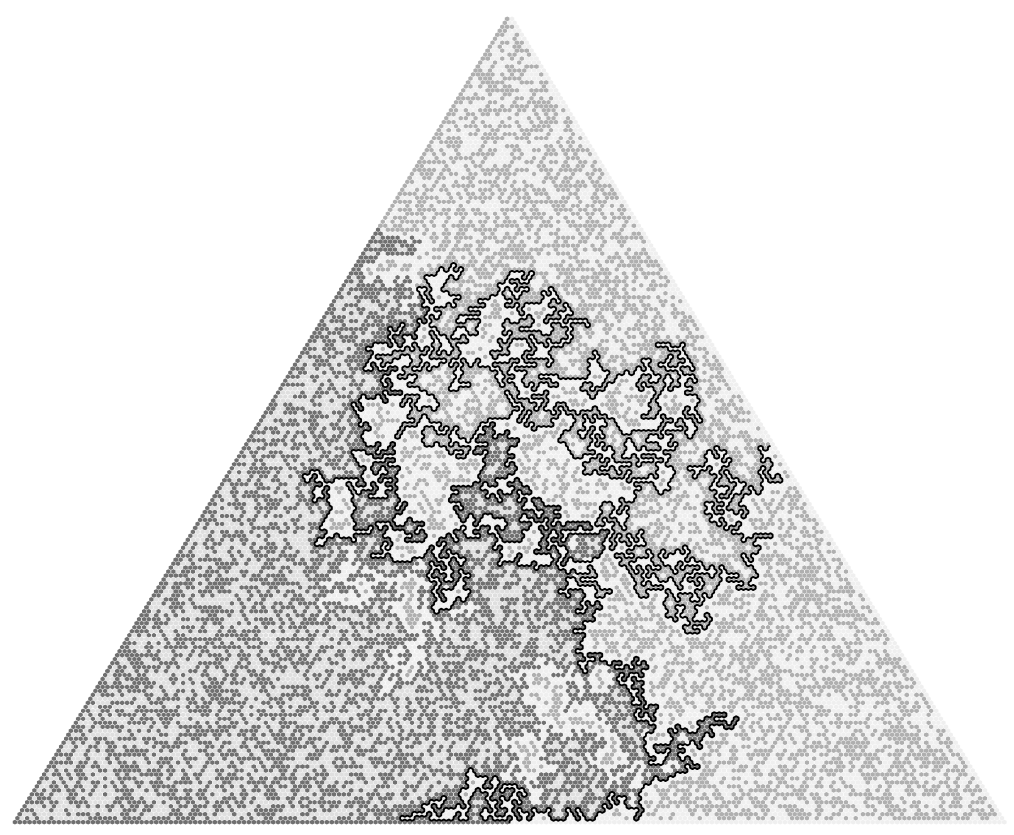

FiguRE 2. A coupling of a near-critical interface with critical percolation.

related to each other by scaling. Let us also mention that near-critical percolation is closely related to the questions concerning the noise-sensitivity of percolation, such as is studied in 26, 12.

Before describing the results of the present paper, let us just emphasize the relevance of such near-critical models for physical applications. Suppose for instance that one is considering a percolation model in a large box, and that one "heats" progressively the system, i.e. that cells become progressively black, one after the other and independently (this corresponds to increase $p$ in the standard coupling described above). Then, we know that the percolation parameter at which a leftto-right crossing of the big box will appear is (with a large probability) close to the critical one. But, if one looks at it more precisely, it is easy to see that the value at which the crossing occurs will be $p^{*}(N, u)$, where $u$ is a uniform random variable in $[-1 / 2,1 / 2]$. Hence, at this moment, the picture and its fine properties are that of near-critical percolation. Another example where this near-critical picture appears spontaneously is that of gradient percolation, an inhomogeneous percolation model introduced in 24] and studied mathematically in 22].

Contributions of this paper. Our focus here will be on the scaling limits of near-critical interfaces. Here is a list of results that we shall derive in the present paper. Choose a sequence $(p(N), N \geq 1)$ and study the behavior of the law of $\Gamma_{N}$ as $N \rightarrow \infty$ :

- For any $\epsilon \in(0,1 / 2)$, if we choose $p(N)$ in such a way that $p(N)$ is close to $p^{*}(N, \epsilon)$, then there exist subsequential limits for the law of $\Gamma_{N}$.

- These limiting laws are all singular with respect to the law of SLE(6). 

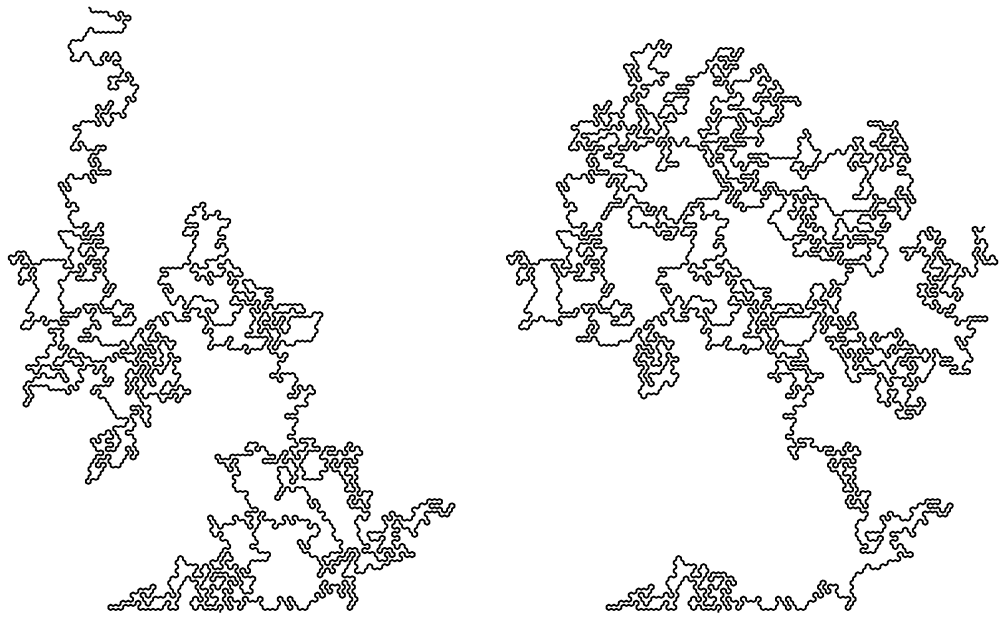

Figure 3. The interfaces at $p_{c}$ and at $p^{*}(N, \epsilon)$ of Figure 2 depicted separately.

- The only other possible subsequential limits for the laws of such curves $\Gamma_{N}$ are the law of an $\operatorname{SLE}(6)$ itself (this is when $p(N)$ is closer to $1 / 2$ ) or a degenerate case where $\gamma$ almost surely follows (the right part of) the boundary of the triangle (this is when $|p(N)-1 / 2|$ is larger).

- The critical exponents associated to these non-degenerate scaling limits are the same as those of SLE(6). In particular, the Hausdorff dimension of the curves is almost surely $7 / 4$.

The first result is a rather direct application of the tightness arguments of Aizenman and Burchard [2] and the definition of the correlation length. The last two statements follow from the ideas of Kesten's paper [16. Our main result is probably the second one, the fact that the laws of non-trivial scaling limits are singular with respect to that of an SLE(6). One way to explain it is the following. In the off-critical regime, one sees on a macroscopic scale a difference between the law of the interface and that of the critical percolation interface (i.e. the non-critical interface is more to the "right" for instance). If one zooms in by a factor $\lambda$, one still sees a difference, but this difference tends to disappear, because one is not looking at a picture of size roughly $L(p)$ any more, but at a picture of size $L(p) / \lambda$. The question is whether this difference disappears sufficiently fast when $\lambda \rightarrow \infty$ or not. Note that one can show that (just as for the critical interface) the number of boxes of size $N / \lambda$ visited by the path is of order $\lambda^{7 / 4}$ when $\lambda$ is large (and $N$ very large). Either this difference vanishes fast with $\lambda$ and one is not able to almost surely detect a difference between the two macroscopic interfaces, or the difference between these two behaviors can be detected by averaging them out over the $\lambda^{7 / 4}$ parts of the path. In the end, one has to compare certain critical exponents to decide which scenario is correct, and it turns out that it is the second one. A "flavor" of supercritical percolation is therefore still present in the scaling limit.

We would like to emphasize that we do not really use any complex analytic SLE technology to derive our results. Our considerations are based on percolation 
techniques, Kesten's scaling ideas and the knowledge of the exact value of the critical exponents (the derivation of which however used SLE).

\section{Preliminaries}

This paper will build on earlier results on near-critical percolation, and in particular on Kesten's paper [16. In this section, we recall some results and ideas that we shall use. All these results are stated and derived in 23. See also the last section of 32 for a rough survey. Throughout the paper, we will use the notation $\asymp$ to say that the ratio between two quantities remains bounded away from 0 and $\infty$. For each $p \in[0,1], P_{p}$ will denote the law of percolation with parameter $p$ (i.e. where each cell/site is black with probability $p$ ).

Recall our definition of the correlation length

$$
L(p)=L(p, \epsilon)=\inf \{N: R(p, N)>1 / 2+\epsilon\} .
$$

Note that $L$ is non-increasing with respect to $p$ and non-decreasing with respect to $\epsilon$.

We define the event $A^{4}(n)$ where there exist four disjoint paths of alternating colors (when ordered clockwise around the origin), each of them connecting a neighbor of the origin to the circle of radius $n$. Similarly, we define the event $A^{2}(n)$ (resp. $A^{1}(n)$ ) where there exist two paths of different colors (resp. one black path) from a neighbor of the origin to the circle of radius $n$. We will use the following properties, that hold for any fixed $\epsilon \in(0,1 / 2)$ :

(1) The Russo-Seymour-Welsh estimates remain valid below $L(p, \epsilon)$ : for all $k \geq 1$, there exists $\delta_{k}(\epsilon)>0$ such that for all $p$, for all $N \leq L(p, \epsilon)$, the probability (for $P_{p}$ ) that there is a black (resp. white) horizontal crossing of a $k N \times N$ parallelogram is at least $\delta_{k}$.

(2) Let $A^{2}\left(n_{1}, n_{2}\right)$ denote the event that there exist two arms of different colors joining the circles of radii $n_{1}$ and $n_{2}$. Then (this follows from the "armseparation lemmas" that we will briefly describe at the end of this section)

$$
P_{p}\left(A^{2}\left(n_{1} / 2\right)\right) \times P_{p}\left(A^{2}\left(2 n_{1}, n_{2}\right)\right) \asymp P_{p}\left(A^{2}\left(n_{2}\right)\right)
$$

uniformly for $p \geq 1 / 2$ and $2 n_{1} \leq n_{2} \leq L(p, \epsilon)$ (this is known as the quasimultiplicativity property). The same is true for four arms and one arm, i.e. with $A^{4}$ or $A^{1}$ instead of $A^{2}$.

(3) It can be used to prove that

$$
\sum_{j=1}^{n} j P_{p}\left(A^{2}(j)\right) \asymp n^{2} P_{p}\left(A^{2}(n)\right),
$$

uniformly for $p \geq 1 / 2$ and $n \leq L(p, \epsilon)$.

(4) We have

$$
P_{p}\left(A^{2}(n)\right) \asymp P_{1 / 2}\left(A^{2}(n)\right) \text { and } P_{p}\left(A^{4}(n)\right) \asymp P_{1 / 2}\left(A^{4}(n)\right)
$$

uniformly for $p \geq 1 / 2$ and $n \leq L(p, \epsilon)$.

(5) Finally,

$$
(p-1 / 2) L(p, \epsilon)^{2} P_{1 / 2}\left(A^{4}(L(p, \epsilon))\right) \asymp 1
$$

as $p \rightarrow 1 / 2^{+}$. 
The last property, which holds for each $\epsilon \in(0,1 / 2)$, implies in particular that for any fixed $\epsilon, \epsilon^{\prime} \in(0,1 / 2)$,

$$
p^{*}(N, \epsilon)-1 / 2 \asymp p^{*}\left(N, \epsilon^{\prime}\right)-1 / 2
$$

as $N \rightarrow \infty$, and that

$$
L(p, \epsilon) \asymp L\left(p, \epsilon^{\prime}\right)
$$

as $p \rightarrow 1 / 2^{+}$. Note that the combination of items (2) and (4) shows that

$$
P_{p}\left(A^{2}\left(n_{1}, n_{2}\right)\right) \asymp P_{1 / 2}\left(A^{2}\left(n_{1}, n_{2}\right)\right)
$$

uniformly for $p \geq 1 / 2,2 n_{1} \leq n_{2} \leq L(p, \epsilon)$ (and similarly for $A^{4}$ ).

Recall also [29] (see also 32]) that the convergence of critical interfaces to $\operatorname{SLE}(6)$ imply that for any $\eta \in(0,1)$,

$$
P_{1 / 2}\left(A^{2}(\eta n, n)\right) \rightarrow f_{2}(\eta) \text { and } P_{1 / 2}\left(A^{4}(\eta n, n)\right) \rightarrow f_{4}(\eta)
$$

as $n \rightarrow \infty$, where $f_{2}(\eta) \sim \eta^{1 / 4+o(1)}$ and $f_{4}(\eta) \sim \eta^{5 / 4+o(1)}$ as $\eta \rightarrow 0^{+}$, which in turn (using the properties of SLE(6)) implies that

$$
P_{1 / 2}\left(A^{2}(n)\right)=n^{-1 / 4+o(1)} \text { and } P_{1 / 2}\left(A^{4}(n)\right)=n^{-5 / 4+o(1)}
$$

as $n \rightarrow \infty$. Analogous statements hold for any number of arms (see [23]).

Note that the correlation length introduced here differs slightly from the usual one - let us denote it here by $L^{*}(p, \epsilon)$ - that is defined in terms of crossing probabilities of rectangles or rhombi (the one introduced in [9] and used, for instance, in [16). Using Russo-Seymour-Welsh considerations (and also the fact that for any $\epsilon, \epsilon^{\prime} \in(0,1 / 2), L^{*}(p, \epsilon) \asymp L^{*}\left(p, \epsilon^{\prime}\right)$; see [16, 23]), these two definitions can easily be shown to be equivalent: for any $\epsilon \in(0,1 / 2), L(p, \epsilon) \asymp L^{*}(p, \epsilon)$. We are thus allowed to use for $L$ results established for $L^{*}$. Alternatively, we could have chosen to work with rhombi instead of triangles, and then we would have used directly the usual definition of correlation length. These two definitions are also known to be equivalent to other "natural" correlation lengths, describing for example the mean radius of a finite cluster or the rate of decay of connectivity properties (see, e.g. the discussion in section 2.2 of [11]). Note also for future reference that $L$ can be increased by at least any constant factor by choosing a larger $\epsilon^{\prime}$ (still by Russo-Seymour-Welsh).

An instrumental technique in the derivation of the quasi-multiplicativity result (and which we shall use in this paper at least three times) are Kesten's "separation lemmas". The precise statements are derived in detail in 23] (see also 32 for a survey); here is a hand-waving description of these ideas in the case of four arms (this is the case that is most relevant for us in the present paper). Suppose for instance that we divide the annulus $\mathcal{A}_{n}$ between the circles of radii $3 n / 4$ and $n$ into 8 slices as depicted in Figure 4, and let $L_{1}, L_{2}, L_{3}$ and $L_{4}$ be four of them that are all at positive distance from each other. Consider percolation on the triangular grid restricted to the disc of radius $n$. Recall that $A^{4}(n)$ is the event where four disjoint arms $u_{1}, \ldots, u_{4}$ of alternating color originate from the neighbors of the origin and reach the unit disc. We define the sub-event $\hat{A}^{4}(n)$ of $A^{4}(n)$, where we add the constraint that for $j=1, \ldots, 4, u_{j} \cap \mathcal{A}_{n} \subset L_{j}$ (see Figure 4). The separation statement states that (uniformly with respect to $n \leq L(p)$ )

$$
P_{p}\left(A^{4}(n)\right) \asymp P_{p}\left(\hat{A}^{4}(n)\right) .
$$



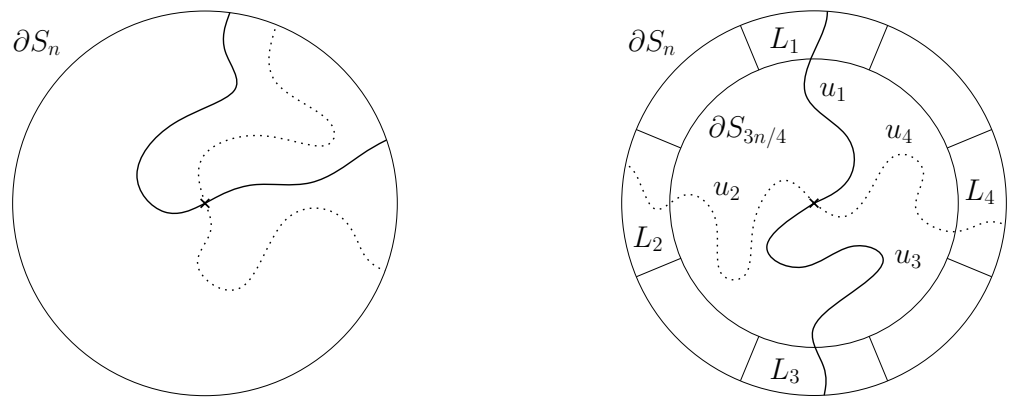

Figure 4 . The events $A^{4}(n)$ and $\hat{A}^{4}(n)$ (sketch).
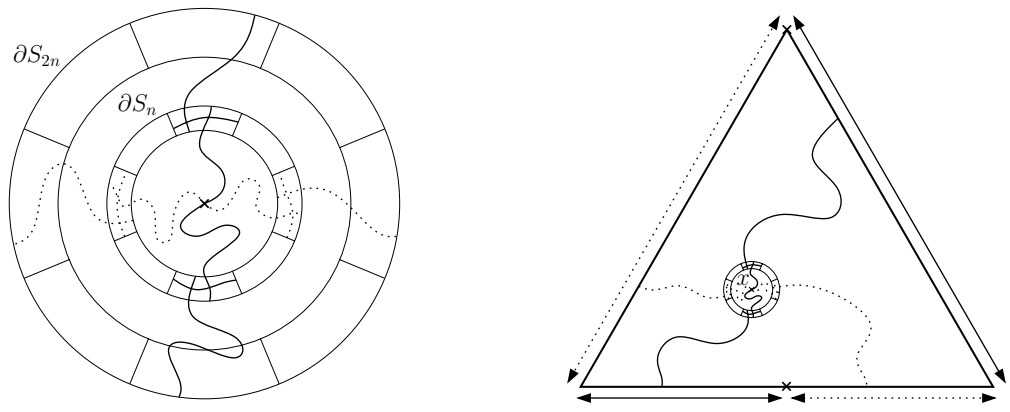

Figure 5. Extension of $\hat{A}^{4}(n)$ into $\hat{A}^{4}(2 n)$, and the pivotal site for the crossing event.

The events $\hat{A}^{4}$ have the nice feature that it is possible (with some care) to "extend" the arms further via Russo-Seymour-Welsh estimates: the conditional probability is positive and bounded from below. For instance (see Figure 5), one gets that

$$
P_{p}\left(\hat{A}^{4}(2 n)\right) \asymp P_{p}\left(\hat{A}^{4}(n)\right) .
$$

More generally, the same argument shows that with positive conditional probability bounded from below, it is possible to extend these four arms into disjoint paths that land on some prescribed boundary parts of a larger domain than the disc (if one considers the percolation in this larger domain).

Since we will be using this type of argument several times, let us briefly describe how this allows us to deduce that

$$
R(p, N)-R(1 / 2, N) \geq c(p-1 / 2) \times N^{2} \times P_{1 / 2}\left(A^{4}(N)\right)
$$

uniformly for $N \leq L(p)$ and $p>1 / 2$. Note that $R(p, N)-R(1 / 2, N)$ is exactly the probability that for some $p^{\prime} \in[1 / 2, p]$, the color of some site $x$ is changed exactly at $p^{\prime}$, and that at that moment, the configuration around $x$ is that of four arms of alternating colors originating at $x$ that join $x$ to prescribed parts of the boundary of the triangle (as in Figure 5). This can happen for only one $x$ and one $p$ for each realization of the coupling. Hence, if we sum only over the sites $x$ that are at a 
distance at least $N / 10$ of the boundary of $T_{N}$ (and there are at least a constant times $N^{2}$ of them) and use the separation lemmas and the fact that the four-arm probabilities are uniformly controlled for $n \leq L(p)$, we obtain the desired lower bound.

\section{Tightness}

For simplicity of presentation, we will stick to the setup that we described in the Introduction, even if the shape of the considered domain (in our case, it is the triangle) could be chosen arbitrarily.

Let us describe this setup more precisely. Here we follow the definitions of [2] and refer the reader to this paper for more details. We consider $T$ the (filled) equilateral triangle of unit side length, with corners $(1 / 2,0),(-1 / 2,0)$ and $(0, \sqrt{3} / 2)$. The rescaled interfaces will be elements of $\mathcal{S}_{T}$, the space of curves in $T$ : these are equivalence classes of continuous functions from $[0,1]$ to $T$, where two functions $f_{1}$ and $f_{2}$ represent the same curve if and only if there exists a continuous increasing bijection $\phi:[0,1] \rightarrow[0,1]$ such that $f_{1}=f_{2} \circ \phi$. We endow this space with the quotient metric:

$$
\Delta\left(f_{1}, f_{2}\right):=\inf _{\phi}\left(\max _{[0,1]}\left|f_{1}-f_{2}\right|\right)
$$

where the infimum is over the set of increasing bijections $\phi$ from $[0,1]$ onto itself.

We call $P_{p, N}$ the law of the rescaled interface $\Gamma_{N}:=\gamma^{N} / N$ of percolation with parameter $p$ in our triangle (with mesh size $1 / N$ ): this is a probability measure on $\mathcal{S}_{T}$. Endowed with the previous metric, $\mathcal{S}_{T}$ is a complete separable space, so that tightness and relative compactness are equivalent (by Prohorov's theorem).

Proposition 1. The family $\left(P_{p, N}, p \in[0,1], N \geq 1\right)$ is relatively compact in the set of probability measures on $\mathcal{S}_{T}$.

Proof. Let us consider a sequence $\left(P_{p_{k}, N_{k}}\right)$ in this family. Our goal is to find a converging subsequence:

- By symmetry, we can assume that $p_{k} \geq 1 / 2$ for all $k \geq 1$.

- If $N_{k}$ remains bounded along a subsequence, proving convergence of a subsequence of $\left(P_{p_{k}, N_{k}}\right)$ is trivial.

- We can therefore restrict ourselves to the case where $N_{k} \rightarrow \infty$. Suppose that for all $\epsilon \in(0,1 / 2), N_{k} / L\left(p_{k}, \epsilon\right) \rightarrow \infty$ along a subsequence. Then it is easy to check (using Russo-Seymour-Welsh arguments) that along that subsequence, $\Gamma^{k}=\Gamma_{N_{k}}$ converges in law to the concatenation of the two parametrized segments $[0,1 / 2] \cup[1 / 2, \sqrt{3} i / 2]$.

- We now suppose that for some $\epsilon \in(0,1 / 2), N_{k} / L\left(p_{k}, \epsilon\right)$ remains bounded. In particular, we get that for some fixed $\epsilon^{\prime}>0, N_{k} \leq L\left(p_{k}, \epsilon^{\prime}\right)$ for all large enough $k$ (since one can increase $L$ by at least any constant factor, by considering $\epsilon^{\prime}$ close enough to $1 / 2$ ). We can then use the machinery developed by Aizenman and Burchard (Theorem 1.2 in [2]): Russo-Seymour-Welsh estimates hold uniformly for all $p$ and $N \leq L\left(p, \epsilon^{\prime}\right)$, so that for any annulus $\mathcal{A}(x ; r, R)=\{z \in T: r<|z-x|<R\}$,

$P_{p_{k}}\left(\mathcal{A}(x ; r, R)\right.$ is traversed by $\left.\Gamma_{N_{k}}\right) \leq P_{p_{k}}\left(A^{1}\left(r N_{k}, R N_{k}\right)\right) \leq C(r / R)^{\alpha}$ 
for two universal constants $\alpha, C$ (here traversed means that it visits the inner and the outer boundary of the annulus). The BK inequality (see, e.g. [14]) then leads to the fact that

$$
P_{p_{k}}\left(\mathcal{A}(x ; r, R) \text { is traversed } K \text { times by } \Gamma_{N_{k}}\right) \leq C_{K}(r / R)^{\alpha K},
$$

which is exactly the hypothesis (H1) of [2] (uniform power bounds on the probability of multiple crossings in annuli).

For the sake of completeness, let us just sketch why tightness can be derived from this. First, the hypothesis (H1) implies regularity properties (Theorem 1.1 in 2]) for the random curves $\Gamma_{N_{k}}$ : for any $\beta>0$, there is some Hölder continuity bound such that for each $k$, there is a probability at least $1-\beta$ that $\Gamma_{N_{k}}$ can be parametrized and satisfy this bound. In particular, we can exhibit some equicontinuous set such that for each $k, \Gamma_{N_{k}}$ belongs to this set with probability at least $1-\beta$. Tightness follows (Theorem 1.2 in [2]) using (a slight adaptation of) Arzela-Ascoli's characterization of compactness for continuous functions on a compact set.

Hence, in all cases, one can find a converging subsequence of $\left(P_{p_{k}, N_{k}}\right)$.

\section{LENGTH AND DIMENSION OF NEAR-CRITICAL INTERFACES}

In this section, we will study the non-degenerate case where $p(N)-1 / 2$ is not too large: we assume that $p(N) \in\left[1 / 2, p^{*}(N, \epsilon)\right]$ for some fixed $\epsilon \in(0,1 / 2)$ (note that this includes the critical case). We exhibit properties that near-critical interfaces share with critical ones. We will first focus on an estimate for discrete interfaces and then study the scaling limits.

4.1. Length of discrete interfaces. The length (number of edges) $\ell\left(\gamma^{N}\right)$ of the discrete interface $\gamma^{N}$ in the near-critical regime is roughly the same as that of the critical interface:

Proposition 2. Assume that $p(N) \in\left[1 / 2, p^{*}(N, \epsilon)\right]$. Then for any fixed $\beta>0$,

$$
\lim _{N \rightarrow \infty} P_{p(N)}\left(\ell\left(\gamma^{N}\right) \notin\left[N^{7 / 4-\beta}, N^{7 / 4+\beta}\right]\right)=0 .
$$

Proof. Note that the hypothesis on $p(N)$ implies in particular that $N \leq L(p(N), \epsilon)$, so that we can use the results on near-critical percolation that we have recalled in the preliminaries.

Let us first derive the upper bound for $\ell\left(\gamma^{N}\right)$ : we estimate its expectation. We note that for any edge $x \in T_{N}$ on the dual hexagonal lattice, $x \in \gamma^{N}$ if and only if there exist two arms as depicted on Figure 6] one black joining a neighbor of $x$ to the negative real half-axis, and one white joining a neighbor of $x$ to the positive real half-axis.

In particular,

$$
P_{p}\left(x \in \gamma^{N}\right) \leq P_{p}\left(A^{2}\left(d\left(x, \partial T_{N}\right)\right)\right) .
$$

It allows us to get an upper bound on $E_{p}\left(\ell\left(\gamma^{N}\right)\right)$ : for each $\beta>0$, and for some constants $c, c^{\prime} \ldots$ (using the estimates on near-critical percolation and the fact that 


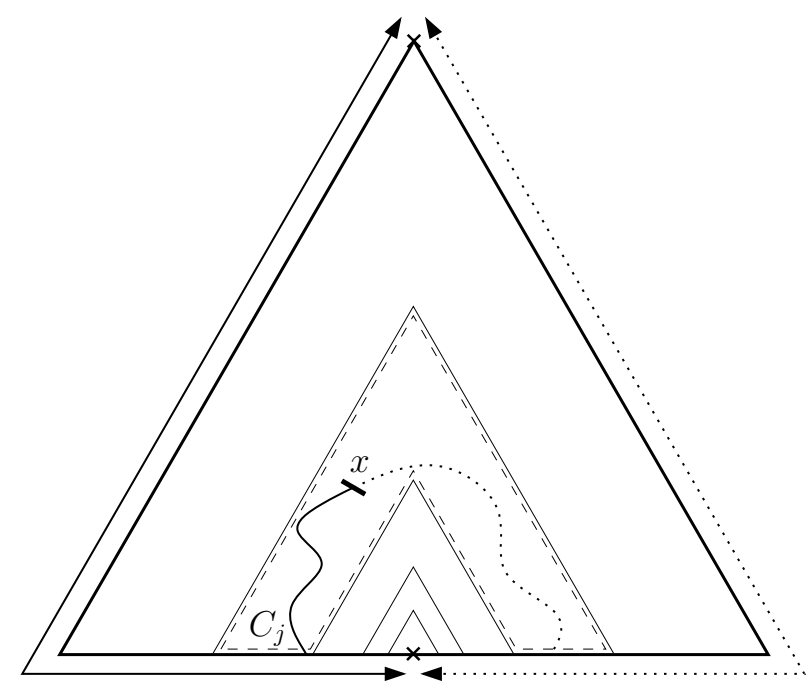

Figure 6. For an edge $x$, being on $\gamma^{N}$ corresponds to the existence of two arms. For the lower bound, we consider semi-annuli $C_{j}=$ $T_{3^{j}} \backslash T_{3^{j-1}}$ and we restrict to the edges at a distance at least $3^{j-3}$ from the boundary of $C_{j}$ (dashed).

there are never more than $3 N$ points at distance $j$ from $\partial T_{N}$ ),

$$
\begin{aligned}
E_{p}\left(\ell\left(\gamma^{N}\right)\right) & \leq \sum_{x \in T_{N}} P_{p}\left(A^{2}\left(d\left(x, \partial T_{N}\right)\right)\right) \\
& \leq c \sum_{x \in T_{N}} P_{1 / 2}\left(A^{2}\left(d\left(x, \partial T_{N}\right)\right)\right) \\
& \leq c^{\prime} \sum_{j=1}^{N} N P_{1 / 2}\left(A^{2}(j)\right) \\
& \leq c^{\prime \prime} N \sum_{j=1}^{N} j^{-1 / 4+\beta / 2} \\
& \leq c^{\prime \prime \prime} N^{7 / 4+\beta / 2} .
\end{aligned}
$$

The upper bound for $\ell\left(\gamma^{N}\right)$ follows immediately by Markov's inequality.

For the lower bound, we use the standard second moment method. For that purpose, take $l$ such that $3^{l} \leq N<3^{l+1}$ and decompose the triangle $T_{N}$ into concentric "semi-annuli" $C_{j}:=T_{3^{j}} \backslash T_{3^{j-1}}, j \leq l$. Note that the curve $\gamma^{N}$ has to cross each of these semi-annuli.

For any edge $x$ in $C_{j}$ at distance at least $3^{j-3}$ from the boundary of $C_{j}$, the results that we have recalled together with the separation lemmas (for two arms) imply that the probability $Q(x)$ that there exist two paths as before, from $\partial x$ to the positive and negative real half-axes, that stay in $C_{j}$, satisfies

$$
Q(x) \asymp P_{1 / 2}\left(A^{2}\left(3^{j}\right)\right)
$$


uniformly for such $x$ and $3^{j} \leq L(p)$. Such an edge $x$ (see Figure 6) is necessarily on $\gamma^{N}$ by the previous remark. Let $\mathcal{N}_{j}$ denote the number of these edges: we have

$$
E_{p}\left(\mathcal{N}_{j}\right) \asymp\left(3^{j}\right)^{2} P_{1 / 2}\left(A^{2}\left(3^{j}\right)\right) \geq\left(3^{j}\right)^{7 / 4-\beta}
$$

for large $j$ (and any fixed $\beta>0$ ).

Now, using the quasi-multiplicativity property and then item (3) we get

$$
\begin{aligned}
E_{p}\left(\mathcal{N}_{j}^{2}\right) & \leq \sum_{x, x^{\prime}} P_{p}\left(A^{2}\left(d\left(x, x^{\prime}\right) / 2\right)\right)^{2} P_{p}\left(A^{2}\left(2 d\left(x, x^{\prime}\right), 3^{j-3}\right)\right) \\
& \leq c E_{p}\left(\mathcal{N}_{j}\right) \times \sum_{k=1}^{3^{j}} k P_{p}\left(A^{2}(k)\right) \\
& \leq c^{\prime} E_{p}\left(\mathcal{N}_{j}\right)^{2} .
\end{aligned}
$$

It follows that (this is the standard "second-moment method")

$$
P_{p}\left(\mathcal{N}_{j} \geq E_{p}\left(\mathcal{N}_{j}\right) / 2\right) \geq \frac{E_{p}\left(\mathcal{N}_{j} 1_{\mathcal{N}_{j} \geq E_{p}\left(\mathcal{N}_{j}\right) / 2}\right)^{2}}{E_{p}\left(\left(\mathcal{N}_{j}\right)^{2}\right)} \geq \frac{E_{p}\left(\mathcal{N}_{j}\right)^{2} / 4}{E_{p}\left(\left(\mathcal{N}_{j}\right)^{2}\right)} \geq \frac{1}{4 c^{\prime}} .
$$

Consider some $j_{0}$ : since the events $\left\{\mathcal{N}_{l-j} \geq E_{p}\left(\mathcal{N}_{l-j}\right) / 2\right\}$ for $j \in\left\{0, \ldots, j_{0}\right\}$ are independent, we get that

$$
P_{p}\left(\mathcal{N}_{l-j}<E_{p}\left(\mathcal{N}_{l-j}\right) / 2 \text { for all } j \leq j_{0}\right) \leq\left(1-1 / 4 c^{\prime}\right)^{j_{0}+1} .
$$

The lower bound follows readily because

$$
\ell\left(\gamma^{N}\right) \geq \max \left(\mathcal{N}_{l}, \mathcal{N}_{l-1}, \ldots\right)
$$

and our lower bound for $E_{p}\left(\mathcal{N}_{j}\right)$.

4.2. Dimension of scaling limits. We now show that scaling limits of nearcritical interfaces have the same Hausdorff dimension as in the critical regime:

Proposition 3. Assume that the law of the curve $\gamma$ is the weak limit of $\left(P_{p_{k}, N_{k}}\right)$, with $N_{k} \rightarrow \infty$ and $p_{k} \in\left[1 / 2, p^{*}\left(N_{k}, \epsilon\right)\right]$. Then the Hausdorff dimension of $\gamma$ is almost surely $7 / 4$.

Similar statements for $n$-point correlation functions, multiple crossings, multifractral spectrum, etc. could be derived in the same way. We leave this to the interested reader (see the informal discussion at the end of the paper) and we focus here only on this fractal dimension.

We might remark that the a priori estimates for the existence of two or three arms near a half-plane or for the existence of five arms in the interior of the domain still hold (uniformly) for $n \leq L(p, \epsilon)$, as these are consequences of the RussoSeymour-Welsh estimates only. Hence, exactly as in the convergence to SLE(6) case (e.g. 28, 32]), the discrete hitting probabilities converge to the continuous hitting probabilities.

Proof. The argument goes along similar lines as for the discrete length. For the upper bound, it suffices to prove that for all $\beta>0$, the expected number of balls of radius $\eta$ needed to cover $\gamma$ is bounded by $\eta^{-7 / 4-\beta}$. But, we know that this expected number is bounded uniformly by this quantity for the discrete paths $\Gamma_{N_{k}}$, and so this also holds in the scaling limit.

For the lower bound, we decompose the triangle into concentric semi-annuli, and we exhibit a family $\left(\mathcal{C}_{j}, j \geq 1\right)$ of independent events of probability at least $c_{0}$, such 
that on each $\mathcal{C}_{j}$, the curve $\gamma$ has dimension $7 / 4$. Again, we follow exactly the same idea (with second moment estimates) as in the discrete case, using the fact (noticed in Section 2) that the discrete estimates for $P_{p}\left(A^{2}\left(n_{1}, n_{2}\right)\right)$ hold uniformly.

\section{The ALternative}

We now prove that for the scaling limits of percolation interfaces, there are only three main options.

Proposition 4. Suppose that $P$ is the weak limit of a sequence $\left(P_{p_{k}, N_{k}}\right)$ when $k \rightarrow \infty$, with $N_{k} \rightarrow \infty$ and $p_{k} \geq 1 / 2$. Then:

- either for each $\epsilon>0, p_{k} \geq p^{*}\left(N_{k}, \epsilon\right)$ for all large $k$, and $P$ is the Dirac mass on the concatenation of the two boundary segments $[0,1 / 2]$ and $[1 / 2, i \sqrt{3} / 2]$,

- or for each $\epsilon>0, p_{k} \leq p^{*}\left(N_{k}, \epsilon\right)$ for all large $k$, and $P$ is the law of an $\operatorname{SLE}(6)$,

- or for some $0<\epsilon^{\prime}<\epsilon<1 / 2, p^{*}\left(N_{k}, \epsilon^{\prime}\right) \leq p_{k} \leq p^{*}\left(N_{k}, \epsilon\right)$ for all large $k$.

Note that these three cases are disjoint. In the last case, it is easy to check that under $P$, the law of the first point on $[1 / 2, i \sqrt{3} / 2] \cup[-1 / 2, i \sqrt{3} / 2]$ that the curve $\gamma$ hits is neither supported on the bottom corners (this is because of $p_{k} \leq p^{*}\left(N_{k}, \epsilon\right)$ ) nor symmetric with respect to the imaginary axis (because $p_{k} \geq p^{*}\left(N_{k}, \epsilon^{\prime}\right)$ ).

Proof. Suppose now that $P$ is the limiting law of a sequence $\Gamma^{k}:=\Gamma_{N_{k}}$ defined under $P_{p_{k}, N_{k}}$, where $N_{k} \rightarrow \infty$ and $p_{k} \geq 1 / 2$.

- Let us first suppose that for each $\epsilon \in(0,1 / 2), N_{k} \geq L\left(p_{k}, \epsilon\right)$ for all sufficiently large $k$. This implies that for each fixed $\epsilon^{\prime}, N_{k} / L\left(p_{k}, \epsilon^{\prime}\right) \rightarrow \infty$ (since we know that the ratio $L(p, \epsilon) / L\left(p, \epsilon^{\prime}\right)$ can be made arbitrarily large, by choosing $\epsilon$ very close to $1 / 2$ ). It follows readily that the probability that there exists a white cluster of radius greater than any fixed positive $u$ for $P_{p_{k}, N_{k}}$ in the rescaled triangle goes to 0 as $k \rightarrow \infty$ and the $P$ must be supported on the path that goes along the right boundary of the triangle.

- Let us now suppose that for each $\epsilon>0, N_{k} \leq L\left(p_{k}, \epsilon\right)$ for all sufficiently large $k$. We assume that the law $P$ is not the law of an SLE(6). The idea is to first translate this asumption in a lower bound for a certain four-arm event, and then, using the facts recalled in section 2 to show that this leads to a contradiction.

In the discrete setting, it is always possible to couple $\Gamma^{k}$ (corresponding to $P_{p_{k}}$ ) with a realization of the interface $\tilde{\Gamma}^{k}$ for $P_{1 / 2}$ in such a way that the sites that are black for the realization of $P_{1 / 2}$ are also black for the realization of $P_{p_{k}}$. In this way, the path $\Gamma^{k}$ is more "to the right" than $\tilde{\Gamma}^{k}$. Letting $k \rightarrow \infty$, we see that we can couple a realization $\Gamma$ of $P$ with an $\operatorname{SLE}(6) \tilde{\Gamma}$ in the triangle $T$ with the same property. We will denote by $\tilde{P}$ the probability measure under which these couplings are defined.

It follows from our results on Hausdorff dimensions (in fact the uniform Russo-Seymour-Welsh bounds would suffice) that $\Gamma$ is almost surely a continuous curve with zero Lebesgue measure. Clearly, one can divide the set of connected components of $T \backslash \Gamma$ into two parts; intuitively speaking, those "to the right of $\Gamma$ " and those "to the left of $\Gamma$ ". Let us call $\mathcal{R}(\Gamma)$ and $\mathcal{L}(\Gamma)$ the respective unions of these components. Similarly, we can define $\mathcal{R}(\tilde{\Gamma})$ and $\mathcal{L}(\tilde{\Gamma})$. Then, we have that $\mathcal{L}(\tilde{\Gamma}) \subset \mathcal{L}(\Gamma)$. 
The fact that the laws of $\Gamma$ and $\tilde{\Gamma}$ are not identical implies that with positive probability the two sets $\mathcal{L}(\tilde{\Gamma})$ and $\mathcal{L}(\Gamma)$ are not equal. It follows (also using the fact that $\Gamma$ is a curve that does not backtrack on its own past) that for some $z \in T$ with rational coordinates and for some positive $r$, if we denote by $D(z, r)$ the disc centered at $z$ with radius $r$,

$$
\tilde{P}(D(z, r) \subset \mathcal{L}(\Gamma) \text { and } D(z, r) \subset \mathcal{R}(\tilde{\Gamma}))>0 .
$$

We can also choose $r$ small enough so that the distance between $z$ and the boundary of the triangle is at least $4 r$. From now on, $z$ and $r$ will be fixed.

The convergence of the discrete interfaces to the continuous ones imply that for some positive constant $c$,

$$
\tilde{P}\left(D(z, 3 r / 4) \subset \mathcal{L}\left(\Gamma^{k}\right) \text { and } D(z, 3 r / 4) \subset \mathcal{R}\left(\tilde{\Gamma}^{k}\right)\right)>c
$$

for any large $k$, with obvious notation. Note that these discrete events are independent of the state of the sites inside $N_{k} D(z, 5 r / 8)$, as they imply that neither $\Gamma^{k}$ nor $\tilde{\Gamma}^{k}$ intersect that disc. On the other hand, the uniform RSW estimates imply that the probability of the event $\mathcal{H}^{k}$ that there exist a white circuit in $N_{k}(D(z, 5 r / 8) \backslash D(z, r / 2))$ for $P_{p_{k}}$ and a black circuit for $P_{1 / 2}$ in the same annulus (where both circuits disconnect $z$ from $N_{k} \partial D(z, 5 r / 8)$ ) is bounded from below (uniformly for all large enough $k$ ) by a positive constant. This event depends only on the state of the sites inside $N_{k} D(z, 5 r / 8)$. Hence, we get that for some absolute positive constant $c^{\prime}$ and all sufficiently large $k$,

$$
\tilde{P}\left(\mathcal{H}^{k} \text { and } D(z, r / 2) \subset \mathcal{L}\left(\Gamma^{k}\right) \text { and } D(z, r / 2) \subset \mathcal{R}\left(\tilde{\Gamma}^{k}\right)\right)>c^{\prime} .
$$

It follows that if one increases continuously $p$ from $1 / 2$ to $p_{k}$ (in the standard coupling), there will exist - with probability bounded from below a value $p$ at which the interface jumps from the left to the right of $D(z, r / 2)$ without hitting it. For this value of $p$, there exist necessarily four arms of alternating colors originating at this site $x$ that is flipped precisely at $p$. The different possibilities are depicted in Figure 7.

For each given site $x$ on the grid $T_{N_{k}} / N_{k}$, let us evaluate the probability that this occurs. When $x$ is not close to the boundary of the triangle, i.e. if it is at distance at least $r / 2$, then we see that four arms of alternating colors must originate from $x$ all the way to distance $r / 2$ from $x$. The probability is then bounded by $P_{p}\left(A^{4}\left(2 N_{k} / r\right)\right) \asymp P_{1 / 2}\left(A^{4}\left(N_{k}\right)\right)$ (recall that $r$ is fixed and that $p \leq p_{k}$ ).

When $x$ is at distance $j / N_{k}<r / 2$ from the boundary, then one must have four arms of alternating colors up to distance $j / N_{k}$, and then two arms that cross a semi-annulus (in a half-plane) from radius $j / N_{k}$ to $r / 2$. Combining this with quasi-multiplicativity and the known bounds (2 arms in a half-plane, 5 arms in the plane that give an upper-bound for the fourarm probability) leads to a bound of the type

$$
c P_{1 / 2}\left(A^{4}\left(N_{k}\right)\right) \times\left(2 j / r N_{k}\right) \times\left(j / N_{k}\right)^{-2+\beta} .
$$

For any $j$, there are at most a constant times $N_{k}$ points on our grid that are at distance exactly $j / N_{k}$ from the boundary of the triangle. Hence, when we sum over all possible sites $x$ in the triangle, we get that the infinitesimal probability that between $p$ and $p+d p$ a site $x$ is flipped in 

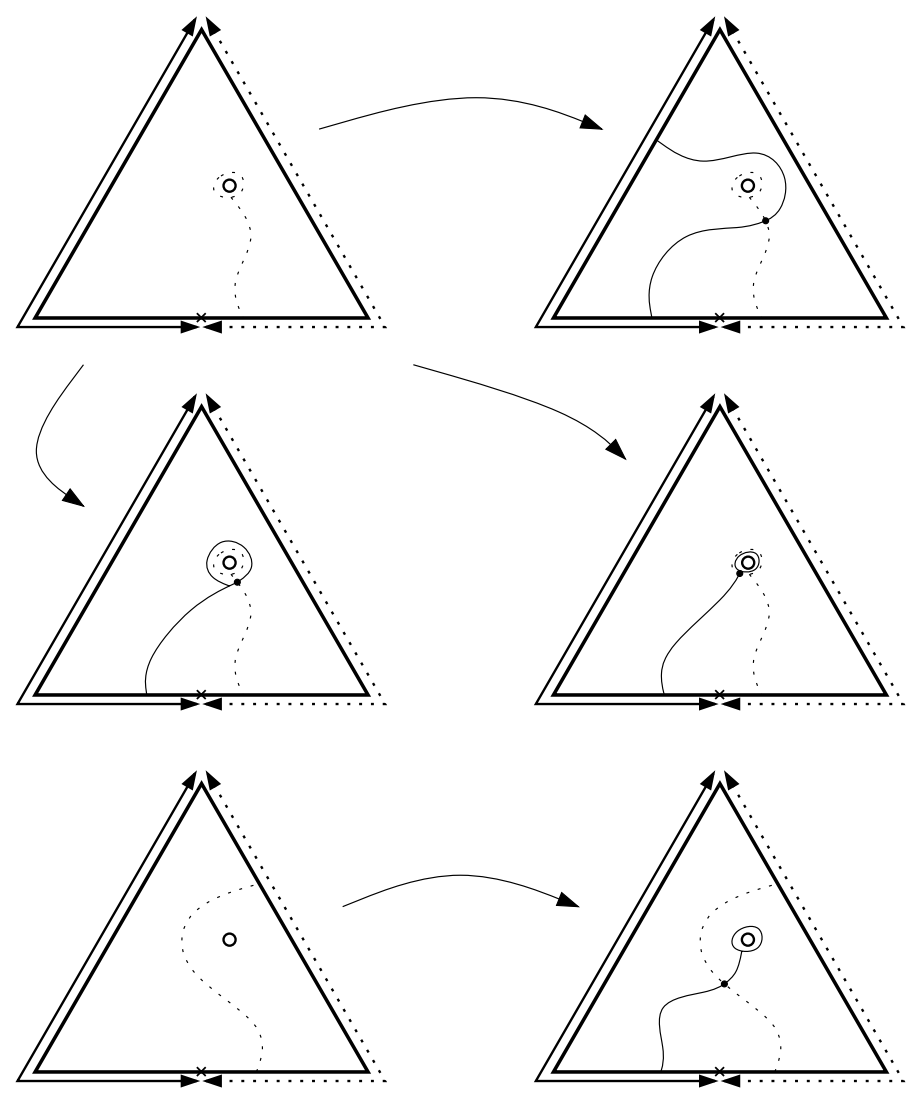

Figure 7 . The different possibilities to jump from the left to the right of the small disc $D(z, r / 2)$.

such a way that the curve jumps from the left to the right of the small disc is bounded by $d p$ times

$c^{\prime} P_{1 / 2}\left(A^{4}\left(N_{k}\right)\right)\left(N_{k}^{2}+N_{k} \times N_{k}^{1-\beta} \sum_{j=1}^{N_{k}} j^{-1+\beta}\right) \leq c^{\prime \prime} N_{k}^{2} P_{1 / 2}\left(A^{4}\left(N_{k}\right)\right)$

(for some constants $c^{\prime}, c^{\prime \prime}$ that do not depend on $k$ ).

It finally follows that for some positive constant $c$,

$$
\left(p_{k}-1 / 2\right) \times N_{k}^{2} \times P_{1 / 2}\left(A^{4}\left(N_{k}\right)\right) \geq c .
$$

We now have shown that the fact that the scaling limit is not an $\operatorname{SLE}(6)$ implies a lower bound for $p_{k}-1 / 2$. But we know (this is the statement recalled at the end of Section 2) that

$$
R\left(p_{k}, N_{k}\right)-R\left(1 / 2, N_{k}\right) \geq c^{\prime \prime \prime}\left(p_{k}-1 / 2\right) \times N_{k}^{2} \times P_{1 / 2}\left(A^{4}\left(N_{k}\right)\right)
$$

for some constant $c^{\prime \prime \prime}$. Hence, we finally get that there exists a positive constant $c^{\prime \prime \prime \prime}$ such that $R\left(p_{k}, N_{k}\right) \geq 1 / 2+c^{\prime \prime \prime \prime}$ for all sufficiently large $k$. In other words, $N_{k} \geq L\left(p_{k}, c^{\prime \prime \prime \prime}\right)$ for all sufficiently large $k$. This contradicts our assumption. 
- Finally to conclude the proof of the proposition, let us suppose that we are not in the last scenario described in its statement, i.e. that it is not true that for some $0<\epsilon^{\prime}<\epsilon<1 / 2, p^{*}\left(N_{k}, \epsilon^{\prime}\right) \leq p_{k} \leq p^{*}\left(N_{k}, \epsilon\right)$ for all large $k$. Then it means that a subsequence of $\left(p_{k}, N_{k}\right)$ falls into one of the two cases that we have just studied. Hence, the limiting probability measure is either the law of SLE(6) or the Dirac mass on the union of the two boundary segments.

\section{ASYMMETRY OF NEAR-CRITICAL INTERFACES}

In this section, we are going to assume that we are in the intermediate regime where the discrete percolation parameter $p$ used at scale $N$ satisfies

$$
p(N) \in\left[p^{*}(N, \epsilon), p^{*}\left(N, \epsilon^{\prime}\right)\right]
$$

for some fixed $0<\epsilon<\epsilon^{\prime}<1 / 2$. The previous alternative tells us that these are the "non-trivial" cases in the large-scale limit. We are going to exhibit asymmetry properties that enable us to detect the difference with a critical interface. We will first derive a result in the discrete setting, and then a result in the continuous setting for the scaling limit.

6.1. Discrete asymmetry. On the discrete level, it is possible to explore the interface dynamically: each time one discovers a new hexagon, one tosses a coin to decide if it is black or white, so that with probability $p$ (resp. $1-p$ ) one makes a 60 degree turn to the right (resp. to the left). Of course, one often bumps into an already discovered hexagon, and in this case the turn is already determined by a previous tossing. Hence, the percolation interface is the image under some map of a sequence of coin tosses. Let us call $\ell^{+}\left(\gamma^{N}\right)$ and $\ell^{-}\left(\gamma^{N}\right)$ the number of black (resp. white) cells neighboring the path $\gamma^{N}$.

Proposition 5. Suppose that $p(N) \in\left[p^{*}(N, \epsilon), p^{*}\left(N, \epsilon^{\prime}\right)\right]$. Then for any fixed $\beta>$ 0 ,

$$
\lim _{N \rightarrow \infty} P_{p(N)}\left(\ell^{+}\left(\gamma^{N}\right)-\ell^{-}\left(\gamma^{N}\right) \geq N^{1-\beta}\right)=1 .
$$

On the other hand, for critical percolation,

$$
\lim _{N \rightarrow \infty} P_{1 / 2}\left(\ell^{+}\left(\gamma^{N}\right)-\ell^{-}\left(\gamma^{N}\right) \leq N^{7 / 8+\beta}\right)=1 .
$$

Proof. Define $v(N)=p(N)-1 / 2$. Clearly the difference between the number of black neighbors discovered by $\gamma^{N}$ and the number of white discovered neighbors evolves like a biased simple random walk with drift $2 v$ (at each step the probability to add one is $1 / 2+v$ ) stopped after a certain random number of steps $\ell$ that is roughly of the order $N^{7 / 4}$ (see Proposition 2). Hence, we see that this difference will be of the order

$$
2 v \times \ell+O(\sqrt{\ell})
$$

(with high probability), i.e. more precisely that for all $\beta>0$,

$$
\lim _{N \rightarrow \infty} P_{p(N)}\left(\ell^{+}\left(\gamma^{N}\right)-\ell^{-}\left(\gamma^{N}\right) \geq 2 v \ell-\ell^{1 / 2+\beta / 2}\right)=1 .
$$

In the off-critical case where $v(N) \geq N^{-3 / 4-\beta / 2}$, we see that the drift term dominates because of Proposition 2 so that the first statement follows readily. Similarly, when $v=0$, the result follows directly from Proposition 2 , 
Hence, the discrete interface in the off-critical regime where $p(N) \geq p^{*}(N, \epsilon)$ will have (on a microscopical scale) anomalously more "black neighbors" than "white neighbors". Moreover, the proof shows that one can detect a good approximation of the actual value of $p(N)$ by looking at this difference because $\left(\ell^{+}\left(\gamma^{N}\right)-\ell^{-}\left(\gamma^{N}\right)\right) / \ell$ will be close to $v(N)$.

But this result is not sufficient to deduce asymmetry of scaling limits. Indeed, if we had for instance taken

$$
p(N)=1 / 2+N^{-b}
$$

for some $b \in(3 / 4,7 / 8)$, then an analogous discrete asymmetry result would hold, i.e. for some small $\beta>0$

$$
\lim _{N \rightarrow \infty} P_{p(N)}\left(\ell^{+}\left(\gamma^{N}\right)-\ell^{-}\left(\gamma^{N}\right) \geq N^{7 / 8+\beta}\right)=1,
$$

but we have proved in the previous section that in this case, the interface still converges to $\operatorname{SLE}(6)$ as $N \rightarrow \infty$ (and we know that $\mathrm{SLE}(6)$ is symmetric).

6.2. Continuous asymmetry. The goal of this section is to prove the following result:

Proposition 6. Suppose that $\gamma$ is the limit (in law) of a sequence of $\Gamma_{N_{k}}$ 's where $p_{k} \in\left[p^{*}\left(N_{k}, \epsilon\right), p^{*}\left(N_{k}, \epsilon^{\prime}\right)\right]$ (and $N_{k} \rightarrow \infty$ ). Then the law of $\gamma$ is singular with respect to that of $S L E(6)$.

Proof. Let us fix a very small positive $\beta$. Define the rectangle $R=[-a, a] \times[0,1 / 4]$ for a small fixed $a$. Define the segments $M=[-a, a] \times\{1 / 8\}$ and $B=[-a, a] \times$ $\{1 / 4\}$, and the smaller triangle $T^{\prime}=\{(x, y) \in T: y \geq 1 / 8\}$ (see Figure 8). For each small equilateral triangle $t$ of size $\eta$ (i.e. that one can obtain by translating $\eta T)$, we define analogously the sets $r, m, b$ and $t^{\prime}$. We say that the triangle $t$ is good for $\gamma$, if at the first hitting time $\sigma(t)$ of $t \backslash r, \gamma(\sigma(t)) \in b$, and if $\gamma$ does exit $r$ through $b$ after its first hitting time of $m$. When there is no ambiguity, we will simply write $\sigma$ instead of $\sigma(t)$.

Let us consider the connected component $d$ of $t^{\prime} \backslash \gamma[0, \sigma]$ that has the top boundaries of $t^{\prime}$ on its boundary. We define the following points on the boundary of $d$ : suppose that we go along the boundary of $d$ clockwise starting from the bottom right corner of $t^{\prime}$ (that we call $a_{0}$ ). Then $a_{1}$ is the right-most point on $m \cap \gamma(0, \sigma)$ and $a_{2}$ is the left-most point on $m \cap \gamma(0, \sigma)$ (see Figure 9). We call $\partial_{1}$ (resp. $\partial_{2}$ ) the part of the boundary of $d$ between $a_{0}$ and $a_{1}$ (resp. between $a_{2}$ and $a_{0}$ ).

When $t$ is a good triangle, we define $F(\gamma, t)$ to be the probability given by Cardy's formula of the existence in $d$ of a black crossing from $\partial_{1}$ to the part $\partial_{3}$ of the boundary between $\gamma(\sigma)$ and $a_{2}$. If after $\sigma, \gamma$ does hit $\partial_{1}$ before $\partial_{2}$, we then say that $t$ is very good for $\gamma$. If $\gamma$ was an $\operatorname{SLE}(6)$, then $F(\gamma, t)$ would just be the conditional probability given $\gamma([0, \sigma])$ that $t$ is very good for $\gamma$.

But here, the law of $\gamma$ is the limit of the law of near-critical percolation interfaces $\Gamma^{k}=\Gamma_{N_{k}}$ defined under $P_{p_{k}, N_{k}}$, where $p_{k} \geq p^{*}\left(N_{k}, \epsilon\right)$ and $N_{k} \rightarrow \infty$. By Skorokhod's representation theorem, we can couple on the same probability space samples of all $\Gamma^{k}$ 's and $\gamma$ in such a way that $\Gamma^{k} \rightarrow \gamma$ almost surely, just as in the proof of convergence of critical percolation interfaces to SLE(6) (as in 32 for instance). For $\Gamma^{k}$, we can also check if a triangle $t$ is good and very good. In fact, for each fixed triangle, the probability that its status for $\gamma$ is not identical to its status for $\Gamma^{k}$ goes to zero as $k \rightarrow \infty$. Indeed, if we suppose for instance that $t$ is 


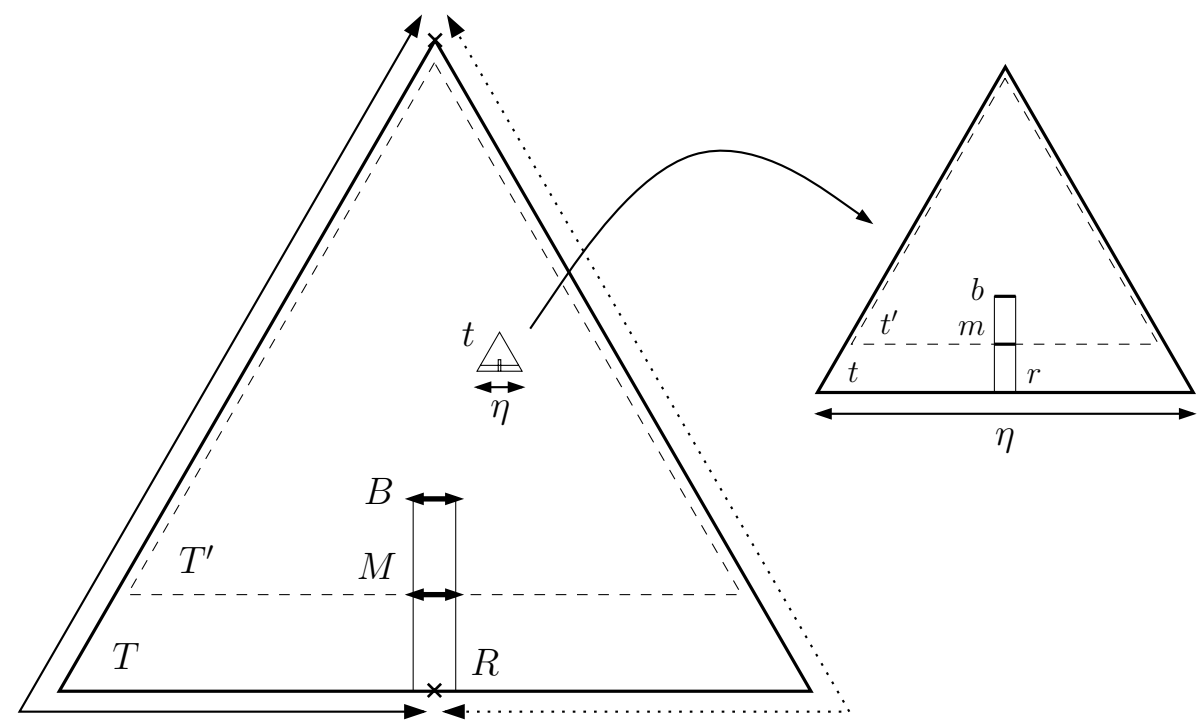

Figure 8 . In the triangle $T$, we consider the subsets $R, M, B$ and $T^{\prime}$. In any small equilateral triangle $t$ of size $\eta$, we similarly define the corresponding sets $r, m, b$ and $t^{\prime}$.

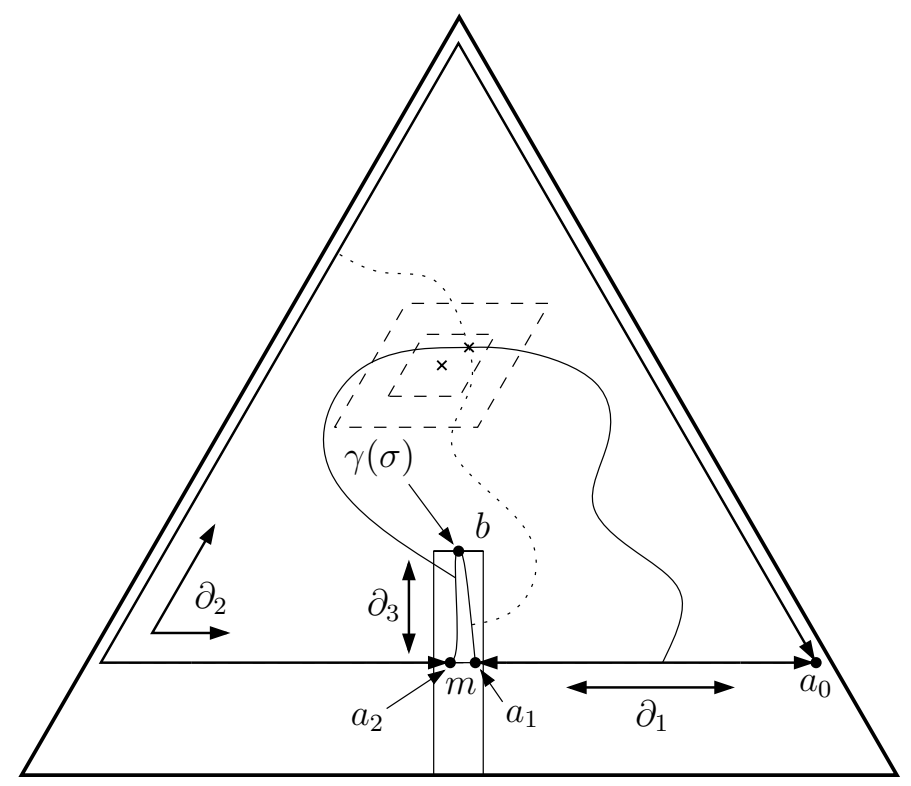

Figure 9. Using "pivotal" sites such as the one depicted here provides a lower bound on the conditional probability that the small triangle $t$ is very good. 
good for $\gamma$ but not for $\Gamma^{k}$, then it means that a three-arm event occurs for $\gamma$ and a priori bounds do exclude this.

If we define the stopping time $\sigma^{k}$ associated to the triangle $t$ and the path $\Gamma^{k}$, this means that when $t$ is good, $\Gamma^{k}\left[0, \sigma^{k}\right]$ converges almost surely to $\gamma[0, \sigma]$. So, the domains $d^{k}$ (with obvious notation) converge in Carathéodory topology towards $d$ almost surely.

We now couple each realization of $\Gamma^{k}$ with a realization $w^{k}$ of critical percolation on the same lattice, in such a way that the realization used to define $\Gamma^{k}$ dominates $w^{k}$ (i.e. its set of black sites is larger). Then (see, e.g. 32]), it follows that the probability $F^{k}\left(\Gamma^{k}, t\right)$ that there exists a crossing from $\partial_{1}^{k}$ to $\partial_{3}^{k}$ in $d^{k}$ for $w^{k}$ (with natural notation) converges almost surely to $F(\gamma, t)$.

Suppose now that the triangle $t$ is good for $\Gamma^{k}$ and consider the configurations as depicted in Figure 9. we restrict ourselves to the case where there exist sites in a smaller rhombus of size comparable to $\eta N_{k}$ from which four arms of alternating colors originate. For each such site $x$, and conditionally on $\Gamma^{k}\left[0, \sigma^{k}\right]$, the probability that this four-arm configuration exists for percolation of parameter $p \in\left[1 / 2, p_{k}\right]$ in the domain not yet explored by $\Gamma^{k}$ is bounded from below by a constant $c$ times $P_{1 / 2}\left(A^{4}\left(\eta N_{k}\right)\right.$ ), uniformly over $p, k$ and the choice of $p$ (this follows again from the uniform estimates for four arms, the separation lemmas and Russo-SeymourWelsh). The probability that this site is flipped from white to black when one increases $p$ from $1 / 2$ to $p_{k}$, and that at this value $p$ the configuration is as described above, is therefore larger than

$$
\left(p_{k}-1 / 2\right) \times c \times P_{1 / 2}\left(A^{4}\left(\eta N_{k}\right)\right) .
$$

Finally, note that this event can happen for only one site (when $p$ increases, since the crossing event is increasing, there can exist only one value of $p$ and therefore one site $x$ such that the state of $x$ is flipped exactly at $p$ and $x$ at that moment is pivotal). Hence, we can sum over all sites $x$ in the small rhombus, and deduce that the conditional probability given $\Gamma^{k}\left[0, \sigma^{k}\right]$ that $t$ is very good is bounded from below by

$$
\begin{aligned}
& F^{k}\left(\Gamma^{k}, t\right)+\left(p_{k}-1 / 2\right) \times c^{\prime}\left(\eta N_{k}\right)^{2} \times P_{1 / 2}\left(A^{4}\left(\eta N_{k}\right)\right) \\
& \quad \geq F^{k}\left(\Gamma^{k}, t\right)+c^{\prime \prime} \eta^{2} \frac{P_{1 / 2}\left(A^{4}\left(\eta N_{k}\right)\right)}{P_{1 / 2}\left(A^{4}\left(N_{k}\right)\right)} \times\left(p_{k}-1 / 2\right) N_{k}^{2} P_{1 / 2}\left(A^{4}\left(N_{k}\right)\right) \\
& \quad \geq F^{k}\left(\Gamma^{k}, t\right)+\frac{c^{\prime \prime \prime} \eta^{2}}{P_{1 / 2}\left(A^{4}\left(\eta N_{k}, N_{k}\right)\right)} \\
& \quad \geq F^{k}\left(\Gamma^{k}, t\right)+\eta^{3 / 4+\beta}
\end{aligned}
$$

(we have used the quasi-multiplicativity to estimate the ratio on the second line) for all small $\eta$ and uniformly with respect to $k$ and to the good configurations $\Gamma^{k}\left[0, \sigma^{k}\right]$. Letting $k \rightarrow \infty$, we conclude that for any good triangle $t$, the conditional probability given $\gamma[0, \sigma]$ that $t$ is very good for $\gamma$ is at least

$$
F(\gamma, t)+\eta^{3 / 4+\beta} \text {. }
$$

We now want to estimate the number of good triangles $t$. We divide the triangle $T$ into $M(\eta) \asymp \eta^{-2}$ small deterministic triangles. Using the same method as when studying the dimension of $\gamma$ (and the arm-separation ideas that allow us to estimate the probability that a triangle is good, i.e. to say that the probability for a triangle $t$ 
to be good is comparable to the probability to be hit), we see that with probability going to 1 as $\eta \rightarrow 0$, the number of good triangles is at least $\eta^{-7 / 4+\beta}$.

We now count how many triangles are very good among the first $\eta^{-7 / 4+\beta} \operatorname{good}$ ones, and we subtract the sum of the $F(t, \gamma)$ for these good triangles. If $\gamma$ was an $\operatorname{SLE}(6)$, the obtained quantity $Z(\eta)$ would satisfy $E(Z)=0$ and $\operatorname{var}(Z) \leq \eta^{-7 / 4+\beta}$. But, in our case, we see that

$$
E(Z) g e \eta^{-7 / 4+\beta} \times \eta^{3 / 4+\beta} \geq \eta^{-1+2 \beta}
$$

and $\operatorname{var}(Z) \leq 4 \eta^{-7 / 4+\beta}$.

If the sequence $\left(\eta_{l}\right)$ has been chosen to converge sufficiently fast to zero, we see that almost surely (using Borel-Cantelli), for all sufficiently large $l$,

$$
Z\left(\eta_{l}\right) \geq \eta_{l}^{-1+3 \beta}
$$

whereas if $\gamma$ had been an $\operatorname{SLE}(6)$, we would almost surely have had

$$
Z\left(\eta_{l}\right) \leq \eta_{l}^{-7 / 8-\beta}
$$

for infinitely many l's.

We thus found an event that holds with probability one for $\gamma$, but with probability zero for SLE(6).

\section{INFORMAL DISCUSSION}

Seemingly, the fact that the laws of near-critical interfaces are singular with respect to the law of SLE(6) surprises some theoretical physicists who work on this topic. Recall that one important aspect of the SLE approach to critical systems was precisely to show that critical conformally invariant models in the same "universality class" give rise to exactly the same curves in the scaling limit. For near-critical models that are not strictly conformally invariant, such as near-critical percolation here, this strong "universality" can fail to be true.

Suppose that a random curve $\gamma$ is the scaling limit of a near-critical percolation interface. The tightness arguments, together with the estimates for existence of multiple arms, indicate that the curve $\gamma$ is almost surely a Loewner chain and that it can be defined via its random driving function $(w(t), t \geq 0)$. It is of course a natural question to ask what this driving function could be (recall that in the case of $\operatorname{SLE}(6), w(t / 6)$ is a standard Brownian motion). Note that after each (fixed or stopping) time, we expect the curve $\gamma$ to turn "more right" than an $\operatorname{SLE}(6)$ would do in the same situation. This leads naturally to the conjecture that (if one extends properly the probability space) one can couple $(w(t), t \geq 0)$ with a standard Brownian motion $(\beta(t), t \geq 0)$ in such a way that $w(t)-\beta(6 t)$ is an increasing continuous process adapted to the filtration generated by the processes $(\beta(6 t))$ and $(w(t))$.

One may think that this contradicts the fact that the law of $(w(t / 6), t \geq 0)$ is singular with respect to that of $\beta$ (since the curves are generated by the driving functions; this is equivalent to saying that the law of $\gamma$ is singular with respect to that of SLE(6)) because the law of Brownian motion with drift is known to be absolutely continuous with respect to that of Brownian motion. But this last fact is only valid under some regularity properties for the drift, and in the present case, one can expect this drift to be quite complicated (its derivative measure might be supported on a set of exceptional times, i.e. a fractal-type set). 
We have seen that important properties (such as the dimension of the curve) are shared by the critical interfaces and the near-critical interfaces in the scaling limit, so that the technology based on conformal invariance of the critical model still provides the correct description of the near-critical interfaces in terms of exponents (this was used in 22] to describe the "percolation front"). In fact, the near-critical percolation technology allows us to prove the following result (this is a direct consequence of the quasi-multiplicativity property and the fact that arm probabilities are comparable uniformly to the arm probabilities at $p=1 / 2$ ): for any $k$, there exists a constant $c_{k}$, such that for any $z_{1}, \ldots, z_{k}$ in $T$, and for any sufficiently small $\epsilon$, the ratio between the probability that $\gamma$ visits the $\epsilon$-neighborhood of these $k$ points and the probability of the same event for $\operatorname{SLE}(6)$ stays in $\left(c_{k}, 1 / c_{k}\right)$ for all small $\epsilon$ and uniformly over the choices of $z_{1}, \ldots, z_{k}$. Loosely speaking, the "finite-dimensional marginals" of the law of $\gamma$ are uniformly comparable to those of SLE(6) (even when the $z_{i}$ 's are very close to each other). Note that this is the type of function that is computable via the Conformal Field Theory technology. But this result does not contradict the fact that the law of $\gamma$ is singular with respect to that of SLE(6). For instance, the constant $c_{k}$ does depend on the number of considered points. Our proof used the fact that we did not zoom on the behavior of the curve at just one point, but that we detected the asymmetry by looking at the mean of the behavior of the curve near more and more points.

\section{ACKNOWLEDGMENT}

We thank Christophe Garban for stimulating discussions and the anonymous referee for careful reading.

\section{REFERENCES}

[1] M. Aizenman, Scaling limit for the incipient spanning clusters, in Mathematics of Multiscale Materials, the IMA Volumes in Mathematics and its Applications 99, Springer (1998). MR.1635999 (99g:82034)

[2] M. Aizenman, A. Burchard, Hölder regularity and dimension bounds for random curves, Duke Math. J. 99, 419-453 (1999). MR1712629(2000i:60012)

[3] C. Borgs, J.T. Chayes, H. Kesten, J. Spencer, The birth of the infinite cluster: finite-size scaling in percolation, Comm. Math. Phys. 224, 153-204 (2001). MR1868996 (2002k:60199)

[4] F. Camia, C.M. Newman, Two-dimensional critical percolation: the full scaling limit, Comm. Math. Phys. 268, 1-38 (2006). MR2249794(2007m:82032)

[5] F. Camia, C.M. Newman, Critical percolation exploration path and SLE(6): a proof of convergence, Probab. Th. Rel. Fields 139, 473-519 (2007). MR2322705

[6] F. Camia, L.R.G. Fontes, C.M. Newman, The scaling limit geometry of near-critical 2D percolation, J. Stat. Phys. 125, 1155-1171 (2006). MR2282484(2007k:82053)

[7] F. Camia, L.R.G. Fontes, C.M. Newman, Two-dimensional scaling limits via marked nonsimple loops, Bull. Braz. Math. Soc. 37, 537-559 (2006). MR2284886

[8] J.T. Chayes, Finite-size scaling in percolation, Proc. ICM 1998 Berlin, Documenta Mathematica, ICM vol. III, 111-122 (1998). MR1648146 (99k:60245)

[9] J.T. Chayes, L. Chayes, J. Fröhlich, The low-temperature behavior of disordered magnets, Comm. Math. Phys. 100, 399-437 (1985). MR802552 (87i:82010)

[10] J.T. Chayes, L. Chayes, D. Fisher, T. Spencer, Finite-size scaling and correlation lengths for disordered systems, Phys. Rev. Lett. 57, 2999-3002 (1986). MR.925751 (89a:82026)

[11] L. Chayes, P. Nolin, Large scale properties of the IIIC for 2D percolation, Stoch. Proc. Appl., to appear.

[12] C. Garban, G. Pete, O. Schramm, The Fourier spectrum of critical percolation, preprint arXiv:0803.3750 (2008).

[13] C. Garban, G. Pete, O. Schramm, in preparation (2008). 
[14] G.R. Grimmett, Percolation, 2nd edition, Springer, New York (1999). MR.1707339 (2001a:60114)

[15] H. Kesten, Percolation theory for mathematicians, Birkhäuser, Boston (1982). MR692943 (84i:60145)

[16] H. Kesten, Scaling relations for 2D-percolation, Comm. Math. Phys. 109, 109-156 (1987). MR879034 (88k:60174)

[17] G.F. Lawler, Conformally invariant processes in the plane, AMS (2005). MR2129588 (2006i:60003)

[18] G.F. Lawler, O. Schramm, W. Werner, Values of Brownian intersection exponents I: Halfplane exponents, Acta Mathematica 187, 237-273 (2001). MR.1879850 (2002m:60159a)

[19] G.F. Lawler, O. Schramm, W. Werner, Values of Brownian intersection exponents II: Plane exponents, Acta Mathematica 187, 275-308 (2001). MR.1879851 (2002m:60159b)

[20] G.F. Lawler, O. Schramm, W. Werner, One-arm exponent for critical 2D percolation, Elec. J. Probab. 7, paper no. 2 (2002). MR1887622 (2002k:60204)

[21] G.F. Lawler, O. Schramm, W. Werner, Conformal restriction: The chordal case, J. Amer. Math. Soc. 16, 917-955 (2003). MR1992830 (2004g:60130)

[22] P. Nolin, Critical exponents of planar gradient percolation, Ann. Probab., to appear.

[23] P. Nolin, Near-critical percolation in two dimensions, Elec. J. Probab., to appear.

[24] B. Sapoval, M. Rosso, J.F. Gouyet, The fractal nature of a diffusion front and relation to percolation, J. Physique Lett. 46, L149-L156 (1985).

[25] O. Schramm, Scaling limits of loop-erased random walks and uniform spanning trees, Israel J. Math. 118, 221-288 (2000). MR.1776084 (2001m:60227)

[26] O. Schramm, J. Steif, Quantitative noise sensitivity and exceptional times for percolation, Ann. Math., to appear.

[27] S. Smirnov, Critical percolation in the plane: conformal invariance, Cardy's formula, scaling limits, C. R. Acad. Sci. Paris Sér. I Math. 333, 239-244 (2001). MR1851632 (2002f:60193)

[28] S. Smirnov, Towards conformal invariance of 2D lattice models, Proc. ICM 2006, vol. 2, 1421-1451 (2007). MR2275653 (2008g:82026)

[29] S. Smirnov, W. Werner, Critical exponents for two-dimensional percolation, Math. Res. Lett. 8, 729-744 (2001). MR1879816 (2003i:60173)

[30] W. Werner, Random planar curves and Schramm-Loewner Evolutions, in 2002 St-Flour summer school, L.N. Math. 1840, 107-195 (2004). MR2079672 (2005m:60020)

[31] W. Werner, The conformal invariant measure on self-avoiding loops, J. Amer. Math. Soc. 21, 137-169 (2008). MR.2350053

[32] W. Werner, Critical two-dimensional percolation, Lecture notes from the IAS/Park City 2007 summer school, preprint arXiv:0710.0856 (2007).

DMA, École Normale Supérieure, 45 rue d'Ulm, 75230 Paris Cedex 05, France, and Laboratoire de Mathématiques, Bât. 425, Université Paris-Sud 11, 91405 Orsay Cedex, FRANCE

E-mail address: pierre.nolin@ens.fr

DMA, École Normale Supérieure, 45 rue d’Ulm, 75230 Paris Cedex 05, France, and Laboratoire de Mathématiques, Bât. 425, Université Paris-Sud 11, 91405 Orsay Cedex, FRANCE

E-mail address: wendelin.werner@math.u-psud.fr 